\title{
A faunistic study on Chalcidoidea (Hymenoptera) of Iran
}

\author{
Hamid Sakenin Chelav ${ }^{1}$, Najmeh Samin ${ }^{2}$, Svetlana N. Myartseva ${ }^{3}$, Shaaban Abd-Rabou ${ }^{4}$, Lütfiye
} GENÇER $^{5}$ \& HAMID NADERIAN 6

${ }^{1}$ Department of Plant Protection, Qaemshahr Branch, Islamic Azad University, Mazandaran, Iran; email: hchelav@yahoo.com

${ }^{2}$ Young Researchers and Elite Club, Science and Research Branch, Islamic Azad University, Tehran, Iran; email: n_samin63@yahoo.com

${ }^{3}$ Cuerpo Académico Entomología Aplicada, Facultad de Ingeniería y Ciencias, Universidad Autónoma de Tamaulipas, 87149, Ciudad Victoria, Tamaulipas, México; email: smyartse@docentes.uat.edu.mx

4Plant Protection Research Institute, Agricultural Research Center, Dokki-Giza, Egypt;

email: shaabanabdrabou59@yahoo.com

${ }^{5}$ Cumhuriyet University, Science Faculty, Department of Biology, 58140, Sivas, Turkey; email: gencer@cumhuriyet.edu.tr

${ }^{6}$ Department of Entomology, Garmsar Branch, Islamic Azad University, Semnan, Iran; email: hamid.naderian@yahoo.com

Sakenin Chelav, H., Samin, N. Svetlana N., Shaaban Abd-Rabou, M., Gençer, L. \& Naderian, H.: $A$ faunistic study on Chalcidoidea (Hymenoptera) of Iran.

Abstract: The fauna of some families of Iranian Chalcidoidea is studied. In total, 24 species of six families, Chalcididae (3 species from 3 genera Brachymeria Westwood, Chalcis Fabricius and Dirhinus Dalman), Encyrtidae (7 species from 6 genera Anagyrus Howard, 1896, Copidosoma Ratzeburg, 1844, Diversinervus Silvestri, 1915, Encyrtus Latreille, 1809, Microterys Thomson, 1876 and Syrphophagus Ashmead, 1900), Eupelmidae (8 species from 4 genera Anastatus Motschulsky, Eupelmus Dalman, Eusandalum Ratzeburg and Pentacladia Westwood), Eurytomidae (3 species from 2 genera Eurytoma Illiger and Tetramesa Walker), Mymaridae (single species from the genus Anaphes Haliday) and Perilampidae (2 species from the genus Perilampus Latreille) were collected and identified.

Keywords: Chalcidoids, Fauna, species diversity, distribution, Iran

\section{Introduction}

Chalcidoid wasps (Hymenoptera: Chalcidoidea) are a fascinating group of insects, which show exquisite life histories and diverse types of host relationships. They are believed to have originated in the upper Jurassic period (NARENDRAN et al. 2007). The superfamily is one of the largest groups of parasitoid wasps with about 22,000 valid species in over than 2,000 genera worldwide (NOYES 2016), with an estimated 500,000 species (HerATY et al. 2013). Although some species are plant feeders or have association with plant galls, but the large majority of chalcid species are primary parasitoids of other insects and arachnids and as such they are important participants in nature's own 
control system for regulating arthropod populations. Over than 800 chalcid species have been associated with targeted biological control programs (GREATHEAD 1986, GODFRAY 1994, Gibson et al. 1997, Bellows \& Fisher 1999).

Chalcidoids' species diversity of Iran was poorly studied so far (MODARRES AwAL 1997) with exception some families - e.g. Aphelinidae (ABD-RABOU et al. 2013), Encyrtidae (FAllahZadeH \& Japoshvili 2010), Eulophidae (TAlebi et al. 2011), Pteromalidae (GHAHARI et al. 2015) and Signiphoridae (GHAHARI et al. 2014). Chalcididae currently includes 87 genera and 1464 species placed in 5 subfamilies in the world. Encyrtidae currently includes 460 genera and 3735 species placed in two subfamilies in the world. Eupelmidae currently includes 45 genera and 907 species placed in 3 subfamilies as follows: Calosotinae, Eupelminae and Neanastatinae. World Eurytomidae currently includes 88 genera and 1424 species placed in three subfamilies: Eurytominae, Heimbrinae, and Rileyinae. Mymaridae currently includes 103 genera and 1424 species in the world. The family Perilampidae currently includes 15 genera and 277 species placed in 3 subfamilies as follows: Chrysolampinae, Perilampinae and Philomidinae (Noyes 2017). The aim of the present study was to survey the major families of Chalcidoidea in some regions of Iran.

\section{Material and methods}

The specimens were collected by sweeping net and Malaise traps from some regions of Iran. The samplings were in 10 provinces, Chaharmahal-Bakhtiari, Golestan, Guilan, Hamadan, Isfahan, Kuhgiloyeh-Boyerahmad, Mazandaran, Qazvin, Semnan and West Azarbaijan (Fig. 1). Also, some parasitoids were obtained through the rearing of their hosts in optimum conditions $\left(27 \pm 1{ }^{\circ} \mathrm{C}, 70 \pm 5 \% \mathrm{RH}, 16: 8 \mathrm{~L}\right.$ : D) in an incubator for emergence of parasitoids. For this purpose, larval stage of some pests in the orders Coleoptera, Hemiptera, Hymenoptera and Lepidoptera were collected and reared. The specimens of this investigation were identified by the authors and some other specialists, and are preserved in the collections of the first and second authors and also some of colleagues named in the acknowledgements. In this paper, classification, nomenclature and distribution were adapted from Noyes (2017).

\section{Results}

In this study 24 species of Chalcidoidea were obtained and identified from different regions of Iran. Chalcis myrifex (Sulzer, 1776) (Chalcididae), Anagyrus belibus (Walker, 1837), Copidosoma peticus (Walker, 1846), Diversinervus elegans Silvestri, 1915, Encyrtus infelix (Embleton, 1902), Microterys chalcostomus (Dalman, 1820), Microterys duplicatus (NeEs, 1834), Syrphophagus herbidus (Dalman, 1820) (Encyrtidae), Eupelmus atropurpureus Dalman, 1820, Eupelmus linearis Förster, 1860, Eupelmus testaceiventris (Motschulsky, 1863), Eusandalum walkeri (Curtis, 1836), Pentacladia eques (Haliday, 1862) (Eupelmidae), Eurytoma onobrychidis Nikol'skaya, 1933, Tetramesa cornuta (Walker, 1832) (Eurytomidae), Anaphes diana (Girault, 1911) (Mymaridae) and Perilampus tristis Mayr, 1905 (Perilampidae) are newly recorded from Iran. The list of species is given below alphabetically with distribution data. 


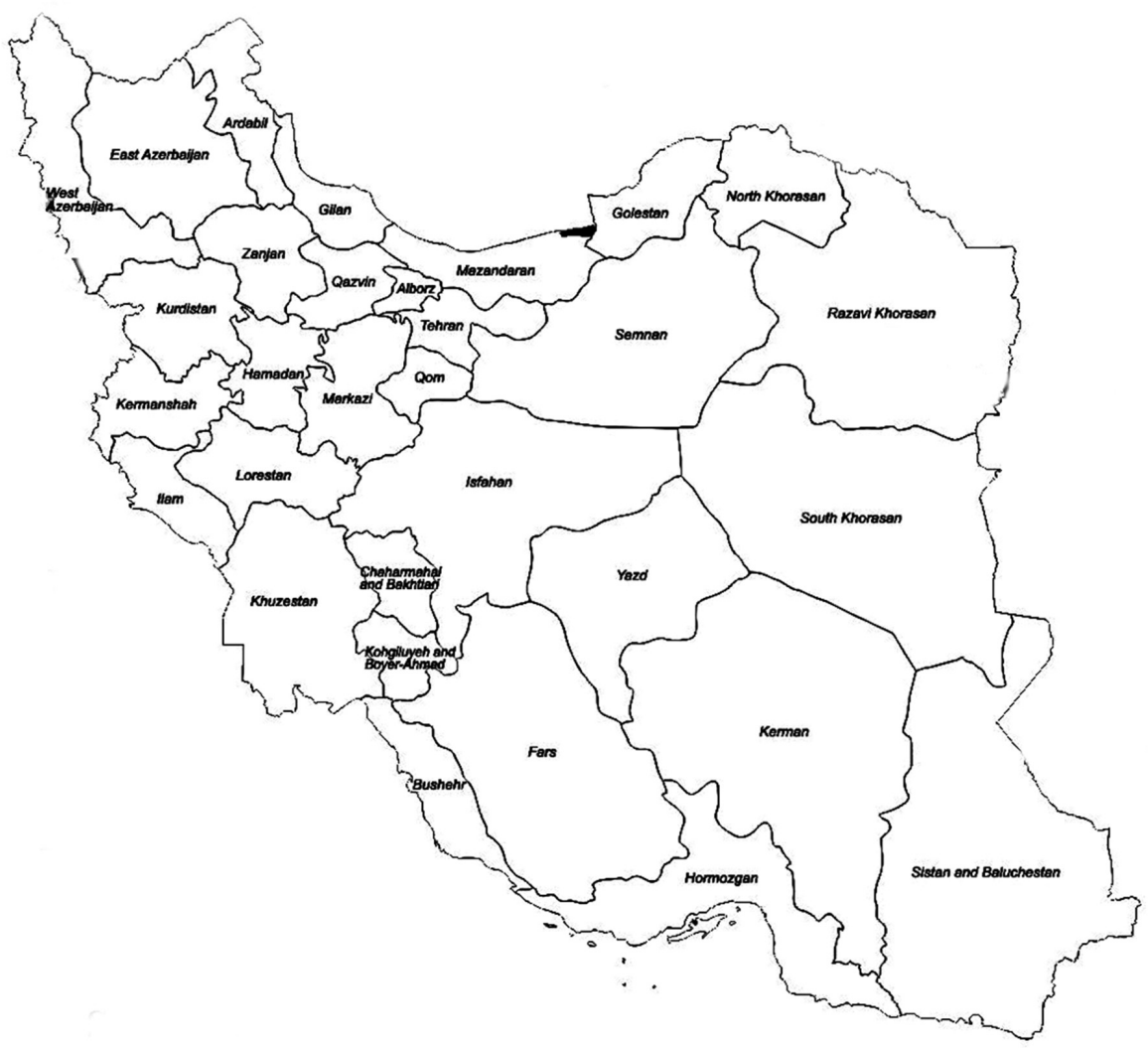

Fig. 1: Map of Iran with provincial boundaries

Family Chalcididae Latreille, 1817

Genus Brachymeria Westwood, 1829

Brachymeria parvula (Walker, 1834)

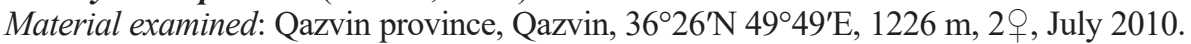

General distribution: Bosnia Hercegovina, Canada, Croatia, Czech Republic, France, Germany, Hungary, Indonesia, Italy, Kazakhstan, Mexico, Moldova, Montenegro, Netherlands, Romania, Russia, Serbia, Siberia, Slovakia, Spain, Sweden, Turkmenistan and USA.

Chalcis myrifex (Sulzer, 1776)

Genus Chalcis Fabricius, 1787

Material examined: Mazandaran province, Savadkooh, $36^{\circ} 05^{\prime} \mathrm{N} 52^{\circ} 55^{\prime} \mathrm{E}, 550 \mathrm{~m}, 1$ 은, August 2014.

General distribution: Croatia, Czech Republic, France, Germany, Hungary, Romania, Russia, Serbia, Slovakia, Spain, Tunisia, Ukraine, UK and USA. 
Genus Dirhinus Dalman, 1818

\section{Dirhinus giffardii Silvestri, 1913}

Material examined: Kuhgiloyeh-Boyerahmad province, Basht, $30^{\circ} 34^{\prime} \mathrm{N} 51^{\circ} 16^{\prime} \mathrm{E}, 878$ m, 1, $1 \hat{\jmath}$, June 2013.

General distribution: Australia, Bolivia, Costa Rica, Dominican Republic, Egypt, Fiji, Hawaii, Israel, Italy, Malawi, Mexico, Micronesia, Nigeria, Pakistan, Peru, Puerto Rico, Trinidad and Tobago, Tunisia and USA.

\section{Family Encyrtidae Walker, 1837}

Genus Anagyrus Howard, 1896

Anagyrus belibus (Walker, 1837)

Material examined: Mazandaran province, Amol, $36^{\circ} 28^{\prime} \mathrm{N} 52^{\circ} 21^{\prime} \mathrm{E}, 2$ ㅇ, $2 \hat{0}^{\hat{}}$, ex Pseudococcus maritimus (Ehrhorn, 1900) (Hemiptera: Pseudococcidae) on Vitis vinifera (Vitaceae), 15.vi.2011.

General distribution: Azerbaijan, Croatia, Czech Republic, Denmark, Europe, Finland, France, Georgia, Germany, Hungary, Lithuania, Madeira, Moldova, Mongolia, Montenegro, Netherlands, Norway, Romania, Russia, Serbia, Spain, Sweden, YK, former USSR.

\section{Copidosoma peticus (Walker, 1846)}

\section{Genus Copidosoma Ratzeburg, 1844}

Material examined: Semnan province, Shahrud, $35^{\circ} 30^{\prime} \mathrm{N} 55^{\circ} 30^{\prime} \mathrm{E}, 1311 \mathrm{~m}, 2$, ex Anthonomus pomorum (Linnaeus, 1758) (Coleoptera: Curculionidae), April 2013.

General distribution: Austria, Bulgaria, China, Czech Republic, Denmark, Europe, Finland, France, Georgia, Germany, Greece, Hungary, Italy, Kazakhstan, Mongolia, Montenegro, Netherlands, Poland, Romania, Slovakia, Spain, Sweden, Switzerland, Turkmenistan, UK, Uzbekistan, former Yugoslavia.

Genus Diversinervus Silvestri, 1915

\section{Diversinervus elegans Silvestri, 1915}

Material examined: Semnan province, Damghan, $35^{\circ} 30^{\prime} \mathrm{N} 54^{\circ} 20^{\prime} \mathrm{E}, 3 \stackrel{\circ}{\circ}, 2 \hat{0^{\lambda}}$, ex Eulecanium rugulosum (Archangelskaya, 1937) on pistachio tree, Pistacia vera (Anacardiaceae), 3.vii.2013.

General distribution: Angola, Argentina, Australia, Brazil, China, Colombia, Cuba, Egypt, Eritrea, Ethiopia, France, Greece, Hawaii, India, Israel, Italy, Kenya, New Caledonia, Morocco, Mexico, Peru, South Africa, Spain, USA, former Yugoslavia.

Genus Encyrtus Latreille, 1809

Encyrtus infelix (Embleton, 1902)

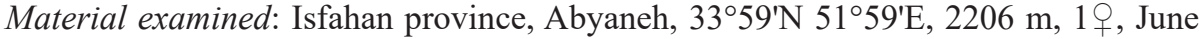
2012. Golestan province, Gorgan, $36^{\circ} 50^{\prime} \mathrm{N} 54^{\circ} 30^{\prime} \mathrm{E}, 2$, ex Coccus hesperidum (Linnaeus, 1758) (Hemiptera: Coccidae) on oleander, April 2014.

General distribution: Algeria, Australia, Austria, Bermuda, Brazil, Colombia, Costa Rica, Cuba, Dominica, Dominican Republic, Ecuador, Egypt, Fiji, French Polynesia, Germany, Hawaii, India, Israel, Jamaica, Kenya, Madagascar, Madeira, Malaysia, Mexico, Morocco, Nearctic, Netherlands, New Caledonia, New Zealand, Peru, Philippines, Puerto Rico, Russia, Seychelles, Sri Lanka, Switzerland, Trinidad \& Tobago, Turkey, UK, United States of America, Venezuela, Vietnam, Zimbabwe. 


\section{Genus Microterys Thomson, 1876}

Microterys chalcostomus (Dalman, 1820)

Material examined: Guilan province, Lahijan, $37^{\circ} 14^{\prime} \mathrm{N} 50^{\circ} 02^{\prime} \mathrm{E}, 2 \circ$, ex Phenacoccus aceris (Signoret, 1875) (Hemiptera: Pseudococcidae) on Fraxinus pensylvanica (Oleaceae), 17.viii.2014.

General distribution: Armenia, Austria, Azerbaijan, China, Czech Republic, France, Georgia, Germany, Hungary, Pakistan, Poland, Slovakia, Spain, Sweden, Ukraine, United Kingdom.

\section{Microterys duplicatus (Nees, 1834)}

Material examined: North Khorasan province, Raz, $37^{\circ} 94^{\prime} \mathrm{N} 57^{\circ} 10^{\prime} \mathrm{E}, 3$, ex Pulvinaria vitis (Linnaeus, 1758) (Hemiptera: Coccidae) on Vitis vinifera (Vitaceae), April 2011.

General distribution: Armenia, Austria, Azerbaijan, Belarus, Czech Republic, Finland, Georgia, Germany, Greece, Hungary, Italy, Moldova, Mongolia, Norway, Poland, Portugal, Russia, Serbia, Slovakia, Spain, Sweden, former Yugoslavia.

\section{Genus Syrphophagus Ashmead, 1900}

\section{Syrphophagus herbidus (Dalman, 1820)}

Material examined: Mazandaran province, Sari, $36^{\circ} 30^{\prime} \mathrm{N} 53^{\circ} 30^{\prime} \mathrm{E}, 2 \circ, 1^{\hat{\jmath}}$, ex Lepidosaphes ulmi (Linnaeus, 1758) (Hemiptera: Diaspididae), August 2012.

General distribution: Armenia, Azerbaijan, Bulgaria, Croatia, Czech Republic, Denmark, Finland, France, Georgia, Germany, Hungary, Moldova, Mongolia, Russia, Slovakia, Sweden, Turkmenistan, UK, former Yugoslavia.

Family Eupelmidae Walker, 1833

Genus Anastatus Motschulsky, 1859

\section{Anastatus japonicus Ashmead, 1904}

Material examined: Guilan province, Siahkal, $36^{\circ} 56^{\prime} \mathrm{N} 49^{\circ} 54^{\prime} \mathrm{E}, 25 \mathrm{~m}, 2$, 1 1 $^{\lambda}$, ex Lymantria dispar (Linnaeus, 1758) (Lepidoptera: Erebidae), June 2014.

General distribution: Austria, Belarus, Bosnia Hercegovina, Bulgaria, Canada, China, Croatia, Czech Republic, France, Germany, Hungary, India, Italy, Japan, Kazakhstan, Kirgizia, Macedonia, Romania, Russia, Serbia, Slovakia, South Korea, Spain, Turkey, Ukraine, USA and Uzbekistan.

Comments: Gypsy moth, Lymantria dispar is one of the most destructive pests in the forsts of northern Iran which many effective parasitoids of Braconidae, Ichneumonidae, A. japonicus and some others decrease its population density.

Genus Eupelmus Dalman, 1820

\section{Eupelmus atropurpureus Dalman, 1820}

Material examined: Mazandaran province, Amol, 36 $28^{\prime} \mathrm{N} 52^{\circ} 21^{\prime} \mathrm{E}, 198$ m, 1 ㅇ, June 2012. General distribution: Austria, Bosnia Hercegovina, Croatia, Czech Republic, Denmark, France, Germany, Hungary, Italy, Kazakhstan, Moldova, Morocco, Netherlands, North Africa, Russia, Serbia, Slovakia, Spain, Sweden, Ukraine, UK and USA.

Eupelmus azureus Ratzeburg, 1844

Material examined: Golestan province, Golestan National Park (Dasht-e-Mirzabaylu, Ghareh-Ghashli), $37^{\circ} 32^{\prime} \mathrm{N} 56^{\circ} 22^{\prime} \mathrm{E}, 1575 \mathrm{~m}, 3 \circ, 1{ }^{\Uparrow}$, ex Andricus fecundatrix (Hartig, 1840), Andricus grossulariae Giraud, 1859 (Hymenoptera: Cynipidae), September 2012.

General distribution: Austria, Bulgaria, Canada, Croatia, Czech Republic, France, Germany, Greece, Hungary, Italy, Moldova, Poland, Romania, Spain, Turkey, Ukraine, UK and USA. 
Eupelmus fulvipes Förster, 1860

Material examined: Mazandaran province, Ramsar, $36^{\circ} 47^{\prime} \mathrm{N} 50^{\circ} 32^{\prime} \mathrm{E}, 9 \mathrm{~m}, 1$, ex

Diplolepis rosae (Linnaeus, 1758) (Hymenoptera: Cynipidae), August 2011.

General distribution: Austria, Azerbaijan, Georgia, Hungary, Italy, Poland, Romania, Russia, Serbia and Spain.

Eupelmus linearis Förster, 1860

Material examined: Guilan province, Rudsar, $36^{\circ} 42^{\prime} \mathrm{N} 50^{\circ} 18^{\prime} \mathrm{E}, 57 \mathrm{~m}, 1$, , September 2010.

General distribution: Croatia, Czech Republic, France, Germany, Hungary, Moldova, Netherlands, Russia, Serbia, Slovakia, Spain and Ukraine.

Eupelmus testaceiventris (Motschulsky, 1863)

Material examined: Razavi Khorasan province, Mashhad, $36^{\circ} 17^{\prime} \mathrm{N} 59^{\circ} 40^{\prime} \mathrm{E}, 996 \mathrm{~m}$, 2 , , August 2011.

General distribution: Australia, China, Croatia, Cyprus, India, Madagascar, Oman, Spain (Canary Islands) and Sri Lanka.

Genus Eusandalum Ratzeburg, 1852

Eusandalum walkeri (Curtis, 1836)

Material examined: Mazandaran province, Babol, $36^{\circ} 30^{\prime} \mathrm{N} 52^{\circ} 35^{\prime} \mathrm{E}, 25 \mathrm{~m}, 1$, , ex Agrilus angustulus (Illiger, 1803) (Coleoptera: Buprestidae), June 2012.

General distribution: Bulgaria, Czech Republic, France, Georgia, Germany, Israel, Romania, Russia, Spain, Ukraine and UK.

Genus Pentacladia Westwood, 1835

Pentacladia eques (Haliday, 1862)

Material examined: Hamadan province, Nahavand, $34^{\circ} 14^{\prime} \mathrm{N} 48^{\circ} 14^{\prime} \mathrm{E}, 1687 \mathrm{~m}, 2$, June 2010.

General distribution: Algeria, Croatia, Egypt, Israel, Mauritania, Niger and Turkmenistan.

Eurytoma bajarii Erdős, 1957

Family Eurytomidae Walker, 1832

Genus Eurytoma Illiger, 1807

Material examined: West Azarbaijan province, Oshnavieh, $37^{\circ} 03^{\prime} \mathrm{N} 45^{\circ} 05^{\prime} \mathrm{E}, 1447 \mathrm{~m}$, 29 , September 2009.

General distribution: France, Hungary, Turkey and former USSR.

Eurytoma onobrychidis Nikol'skaya, 1933

Material examined: Chaharmahal-Bakhtiari province, Lordegan, $31^{\circ} 26^{\prime} \mathrm{N} 50^{\circ} 50^{\prime} \mathrm{E}$, 1609 m, 1, April 2009.

General distribution: Bulgaria, Canada, Czech epublic, Europe, Germany, Hungary, Kirgizia, Slovakia, Sweden, Turkey and Ukraine.

Genus Tetramesa Walker, 1848

Tetramesa cornuta (Walker, 1832)

Material examined: Mazandaran province, Qaemshahr, $36^{\circ} 28^{\prime} \mathrm{N} 52^{\circ} 52^{\prime} \mathrm{E}, 8 \mathrm{~m}, 1^{\circ}$, 20̂, July 2010.

General distribution: Bulgaria, Czech Republic, Hungary, Kazakhstan, Moldova, Romania, Slovakia, Sweden, Turkey, Ukraine, UK, USA and Yemen. 
Family Mymaridae Haliday, 1833

Genus Anaphes Haliday, 1833

Anaphes diana (Girault, 1911)

Material examined: Golestan province, Minudasht, $37^{\circ} 10^{\prime} \mathrm{N} 55^{\circ} 30^{\prime} \mathrm{E}, 78 \mathrm{~m}, 1$, August 2009.

General distribution: Algeria, Australia, Austria, Belgium, Bulgaria, Canada, France, Germany, Greece, Italy, Moldova, Morocco, New Zealand, Romania, Spain, Syria, Turkey, UK and USA.

Family Perilampidae Förster, 1856

Genus Perilampus Latreille, 1809

Perilampus auratus (Panzer, 1798)

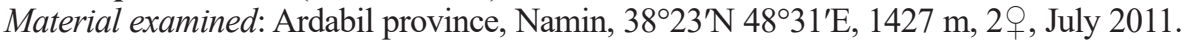

General distribution: Croatia, Czech Republic, Germany, Hungary, Kazakhstan, Moldova, Netherlands, Russia, Slovakia, Sweden and Ukraine.

Perilampus tristis Mayr, 1905

Material examined: Mazandaran province, Nur, $36^{\circ} 19^{\prime} \mathrm{N} 52^{\circ} 00^{\prime} \mathrm{E}, 8 \mathrm{~m}, 2$, ex Zeuzera pyrina (Linnaeus, 1761) (Lepidoptera: Cossidae), June 2012.

General distribution: Argentina, Austria, Belgium, Canada, China, Croatia, Czech Republic, France, Germany, Hungary, Iraq, Israel, Italy, Kazakhstan, Lebanon, Moldova, Mongolia, Netherlands, Romania, Russia, Serbia, Slovakia, Sweden, Switzerland, Syria, Ukraine, UK and USA.

\section{Discussion}

Most of the collected species in this research are parasitoids of agricultural pests and can be efficient biological control agents. Among the 24 recorded species, 14 species were collected from northern Iran, Golestan, Guilan and Mazandaran provinces (south of Caspian Sea); since these areas contain vast forests, various crop fields and ornamental, so these beneficial insects can be effective in biological control of various pests. Conservation of these natural enemies wi+ll result to decreasing of pesticides application in different agroecosystems (FLINT \& DREISTADT 1998). Determining of the hosts of these parasitoids is really important subject which must be attended in order to establishment of classical and applied biological control programs. The fauna of Iranian Chalcidoidea was studied rather well in several contributions. Among the 20 families of Chalcidoidea (Noyes 2017) thirteen of Iranian chalcidoids, Agaonidae (with two species: GHahari \& VAN NoORT 2011), Aphelinidae (with 138 species: ABD-Rabou et al. 2013), Chalcididae (with 42 species: LotFALIZADEH et al. 2012 - plus single new record in this work), Encyrtidae (with 180 species: FALlAHZADEH \& JAPOSHVILI 2017; GUERRIERI \& GHAHARI 2018 - plus seven new records in this work), Eulophidae (with 139 species: GHAHARI 2015), Eupelmidae (with 31 species: LotFaLIZADEH \& GHADIRZADEH 2016 - plus five new records in this work), Eurytomidae and Torymidae (with 37 and 80 species respectively: StOJANOva \& GHAHARI 2009; FAllaHZADEH et al. 2009; GHahari \& Doganlar 2017 - plus two new records of Eurytomidae in this work), Leucospidae (with four species: LOTFALIZADEH \& FAKHRZADEH, 2012), Mymaridae (with 10 species: SAmIN 2015 - plus one new record in this work), Perilampidae (with two species: SAMIN 2015 - plus one new record in this work), Pteromalidae (with 227 species: GHAHARI et al. 2015), Signiphoridae (with 11 species: GHAHARI et al. 2014), and 
Trichogrammatidae (with nine species: ModARRES AwAL 1997) were catalogued. Thus, with this research total number of species of Iranian Chalcidoidea has been reached to 876 (Fig. 2); of course since some families have not been studied perfectly so far, the mentioned number will be increased after updating the related checklists and new faunistic surveys. Conducting faunistic surveys on different families of Iranian Chalcidoidea is necessary for completing the list of Iranian species diversity. Nevertheless, very little attention has been paid till now to the hosts of the Iranian Chalcidoidea. In this research, in addition to the new records several other specimens were collected which are under identification.

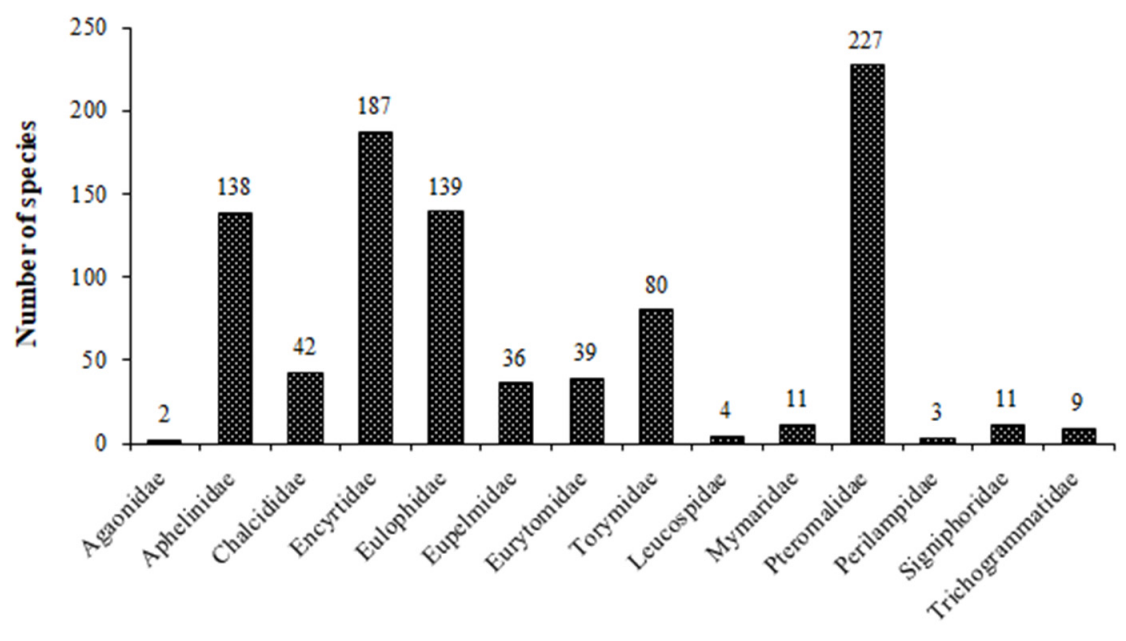

Families of Chalcidoidea

Fig. 2: Species diversity of the families of Iranian Chalcidoidea

\section{Acknowledgements}

The authors are grateful to V.A. Trjapitzin (Russia), M. Doğanlar (Turkey), N.S. Gadallah (Egypt), the late A. Ribes (Spain), H. Ghahari (Iran), M. Hayat (India) and E. Ruíz Cancino (Mexico) for their help in identification of some materials, providing some papers and editing the manuscript. The research was supported by Islamic Azad University (Qaemshahr Branch, and Young Researchers and Elite Club, Science and Research Branch), and Agricultural Research Center (Egypt). 


\section{References}

Abd-Rabou, Sh., Ghahari, H., Myartseva, S.N. \& Ruíz-Cancino, E. 2013: Iranian Aphelinidae (Hymenoptera: Chalcidoidea). - Journal of Entomology and Zoology Studies 1(4): 116-140.

Bellows, T. S. \& Fisher, T. W. 1999: Handbook of biological control. - Academic Press, San Diego, CA, 1046 pp.

Fallahzadeh, M., Narendran, T. C. \& Saghaei, N. 2009: Insecta, Hymenoptera, Chalcidoidea, Eurytomidae and Torymidae in Iran. - Check List 5: 830-839.

FallahzadeH, M. \& Japoshvili, G. 2017: An updated checklist of Iranian Encyrtidae (Hymenoptera, Chalcidoidea). - Zootaxa, 4344 (1): 1-46.

Flint, M. L. \& Dreistadt, S. H. 1998: Natural enemies handbook, the illustrated guide to biological control. - University of California Press, Berkeley, CA, 154 pp.

GHAhari, H. 2015: A contribution to the study of Eulophidae (Hymenoptera: Chalcidoidea) from Iran. - Natura Somogyiensis 27: 55-62.

Ghahari, H. \& VAN NoORT, S. 2011: A comment on Iranian fig wasps (Chalcidoidea: Agaonidae, Pteromalidae). - Linzer biologische Beiträge 43/2: 1247-1252.

Ghahari, H., Myartseva, S.N., Huang, J., Ruiz-Cancino, E. \& Abd-Rabou, Sh. 2014: A checklist of the Iranian Signiphoridae (Hymenoptera: Chalcidoidea). - Wuyi Science Journal 30: 74-82.

Ghahari, H., Doganlar, M., Sureshan, P.M. \& Ostovan, H. 2015: An annotated catalogue of the Iranian Pteromalidae (Hymenoptera: Chalcidoidea). - Entomofauna (Supplement) 19: 1-101.

Ghahari, H. \& Doganlar, M. 2017: An annotated catalog of the Iranian Torymidae (Hymenoptera: Chalcidoidea). - Transactions of the American Entomological Society 143: 453-472.

Gibson, G. A. P., Huber, J. T. \& Woolley, J. B. 1997: Annotated keys to the genera of Nearctic Chalcidoidea (Hymenoptera). - National Research Council of Canada Research Press, Ottawa, Canada, 794 pp.

GodfraY, H. C. J. 1994: Parasitoids, behavioral and evolutionary ecology. - Princeton University Press, 473 pp.

Greathead, D. J. 1986: Parasitoids in classical biological control, pp. 289-318. In: Waage, J. \& Greathead, D. (eds). Insect Parasitoids. - Academic Press, London, 389 pp.

GUERRIERI, E. \& GHAHARI, H. 2018: New records, descriptions and notes on Encyrtidae (Hymenoptera: Chalcidoidea) from Iran. - Zootaxa 4444 (3): 316-326.

Heraty, J. M., Burks, R. A., Cruaud, A., Gibson, G. A. P., Liljeblad, J., Munro, J., Rasplus, J.-Y., Delvare, G., Jansta, P., Gumovsky, J., Huber, J., Woolley, J.B., Krogmann, L., Heydon, S., Polaszek, A., Schmidt, S., Darling, D. C., Gates, M. W., Murray, E., Molin, A. D., Triapitsyn, S., Baur, H., Pinto, J. D., Van Noort, S., George, J. \& Yoder, M. 2013: A phylogenetic analysis of the megadiverse Chalcidoidea (Hymenoptera). - Cladistics 29: 466-542.

Lotfalizadeh, H. \& Fakhrzadeh, N. 2012. A short review of the family Leucospidae (Hym.: Chalcidoidea) in Iran. - Biharean Biologist 6(1): 51-54.

Lotfalizadeh, H., Ebrahimi, E. \& Delvare, G. 2012: A contribution to the knowledge of family Chalcididae (Hymenoptera: Chalcidoidea) in Iran. - Journal of Entomological Society of Iran 31(2): 67-100.

Lotfalizadeh, H. \& GHadirzadeH, L. 2016: Review of Iranian Eupelmidae (Hymenoptera: Chalcidoidea), with five new records. - Journal of Insect Biodiversity and Systematic 2(2): 181-192.

Modarres Awal, M. 1997: Hymenoptera, pp. 260-285. In: Modarres Awal, M. (ed.), List of agricultural pests and their natural enemies in Iran. - Ferdowsi University Press, 429 pp.

Narendran, T. C., Santhosh, S. \& Sudheer, K. 2007: Biosystematics and biogeography of Oriental Chalcidoidea (Hymenoptera) associated with plant galls. - Oriental Insects 41: 141-167.

NoYeS, J.S. 2017: Universal Chalcidoidea Database. World Wide Web electronic publication. - http://www. nhm.ac.uk/chalcidoids (Accessed: 28 December 2016).

Samin, N. 2015: A faunistic study on some families of Chalcidoidea (Hymenoptera) from Iran. - Arquivos Entomoloxicos 14: 119-124.

Stojanova, H. \& Ghahari, H. 2009: Checklists of Iranian Eurytomidae and Torymidae (Hymenoptera, Chalcidoidea). - Linzer biologische Beiträge 41/1: 845-862.

Talebi, A. A., Mohammadi Khoramabadi, A. \& Rakhshani, E. 2011: Checklist of eulophid wasps (Insecta: Hymenoptera: Eulophidae) of Iran. - Check List 7(6): 708-719. 
